\title{
Controlled Magnetic Devices in Power Electronic Applications
}

\author{
Peter Zacharias, Thiemo Kleeb, Florian Fenske, Jiajing Wende, Jonas Pfeiffer \\ UNIVERSITY OF KASSEL \\ Department of Electrical Power Engineering (EVS) \\ Wilhelmshöher Allee 71 \\ 34121 Kassel, Germany \\ Tel.: +49 / (0)561 - 8046344 \\ Fax: +49 / (0)561 - 8046521 \\ E-Mail: peter.zacharias@uni-kassel.de \\ URL: http://www.evs.uni-kassel.de
}

\section{Acknowledgements}

Parts of the work presented in this paper have been supported by the German Federal Ministry for Economic Affairs and Energy (BMWi). Project funding reference number: 0324106A. Responsibility for the contents of this publication lies with the authors.

\section{Keywords}

«Magnetic device», «Energy Control Unit (ECU)», «Passive component», «Device characterisation», «Device Modelling»

\begin{abstract}
Almost all power electronic converters require magnetic devices and some properties of the converters can be controlled with magnetics. New magnetic materials enable implementation of magnetic control of converters also in high switching frequency applications. The presented paper gives an overview about controllability of magnetic devices in theory and practice, simulation approaches and examples. The focus is on electrically controllable magnetic devices. This means that an additional control winding is wound on or integrated into the magnetic core of the device. This additional winding is used to control the inductive behavior of the device by injecting a current.

The different basic control approaches of magnetic devices are explained theoretically and demonstrated experimentally by laboratory prototypes. Several controllable magnetic devices were tested in order to evaluate the applicability of the different control methods. The control techniques were demonstrated also with different core materials to address different applications. Especially ferromagnetic alloys and ferrite materials are in focus of the investigation. Moreover, first approaches for the modelling and simulation of controllable magnetic devices are given in the paper.
\end{abstract}

\section{Introduction}

In comparison to semiconductor devices, magnetic devices have much higher robustness related to overload conditions or ruggedness related to cosmic radiation. The design of magnetic devices represents an important degree of freedom in power electronic systems design. In the past, before 1980, magnetically controlled functions in power electronic converters were quite common [1]. Then, attention of converter designers focused on the promising fast controllability of semiconductor devices. Meanwhile many new hard and soft magnetic materials have been developed offering a lot of interesting options of additional or complementary control for power electronic circuits. This paper presents an overview of these options.

The implementation of special L(I) characteristics of inductors by means of a special shape of the air gap or insertion of ferromagnetic sheets into the air gap are well known. Since about 10 years, another option is the insertion of permanently pre-magnetized sheets into the air gap, also for HF 
applications [2]. This technique became applicable by means of plastic bounded high coercive permanent magnetic powder materials. In both cases the change of the L(I) characteristic is enabled by mechanical measures. This article is focused on electrically controlled devices. Subsequent design examples and approaches for modelling of the devices for effective simulation in electronic circuits with selected applications are presented.

\section{Basic control approaches of inductive devices}

There are different approaches to control magnetic devices electrically. One effective method is DC biasing. The magnetization characteristic is changed by means of a direct current bias of predefined magnetic sections. The total reluctance of the entire magnetic core can be adapted in order to change the inductance value of the component. This will change the reactance of the component and, therefore, influences the electric circuit. This measure enables also the control of electric circuits and converters. Subsequent three methods of DC bias magnetization of inductors are presented.

- Parallel DC biasing: One of the possibilities to change the inductance of an inductor by DC biasing is to generate a magnetic control flux parallel to the magnetic main flux. In this case, both magnetic fluxes are either aligned in the same or opposing direction. Fig. 1 a) shows an example for parallel biasing of an inductor branch. The advantage of this method is the direct influence of the control flux on the reluctance of the core. Even low excitations in the control winding can enable significant reluctance changes. The disadvantage is the coupling between the main winding and the control/biasing winding. AC current flow in the main winding can induce an unwanted voltage drop in the biasing winding and vice versa. Induced AC signals have to be suppressed by filter measures in order to ensure a proper biasing. E.g. [3] implemented this biasing method for the core loss measurement of toroids under DC bias conditions.

Another possibility is to branch the magnetic flux. This means the main winding and the biasing winding are not placed on the same limb of the core as it is presented in [4] or [5]. This measure weakens the influence of the bias flux on the main flux. In turn, the coupling between both windings is reduced and the issue of the induction of unwanted voltage drops is reduced respectively.

- Bias winding parallel to the main flux: A further method to control an inductor by DC biasing is to generate the magnetic control flux orthogonal to the magnetic main flux, such as shown in Fig. 1 b). The control winding is placed through a hole in the core. The magnetic control flux is orthogonal to the main flux and inhomogeneous, because its magnetic field strength depends on the distance from the center axis. This approach offers the advantage that there is no magnetic coupling between main and biasing winding. Therefore, the change in the inductance by this method does not have any retroactive effects on the current in an electric circuit. A significant change of the reluctance requires high ampere turns introduced by the biasing winding.

- Bias winding orthogonal to the main flux: Fig. $1 \mathrm{c}$ ) shows another DC biasing method for controlling the inductance of an inductor. Compared to the previous method, the DC control winding is arranged orthogonal to the main magnetic flux $\Phi 1$. The DC bias current can be used to saturate a region of the core. This has the effect of a variable virtual air gap (VAG), which makes the reluctance of the entire core controllable. Due to the orthogonal direction of the biasing winding to the main flux, the bias flux will enforce the main flux on one side of the bias winding and lower the main flux on the other side. 


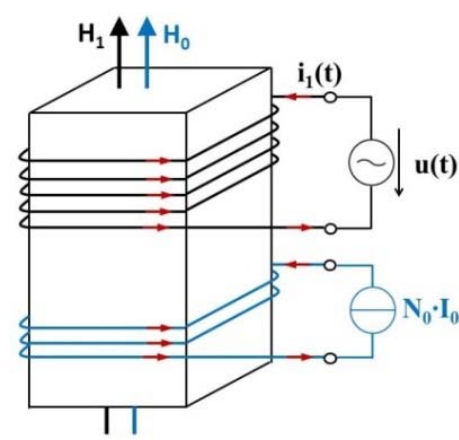

a)

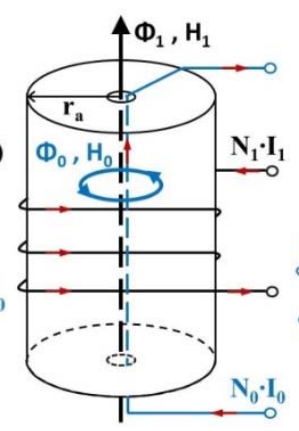

b)

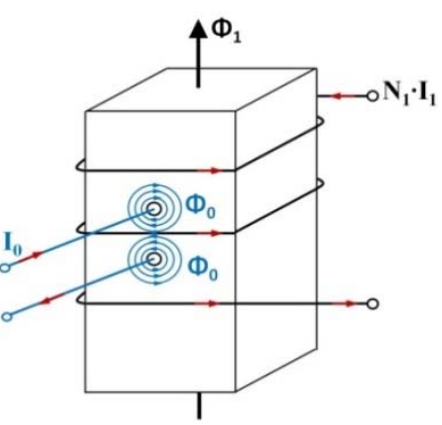

c)

Fig. 1: a) Inductor branch with DC bias winding. b) Orthogonal DC bias with inhomogeneous magnetic control flux. c) DC bias with an orthogonal DC control winding

\section{Bias winding orthogonal and parallel to the main flux}

\section{Controlled devices based on ferrous iron alloys}

Many examples are known from literature, using DC biased magnetic devices based on metallic sheet cores. Novel materials with high permeance and good HF performance make new approaches interesting, even for higher power applications. As first order approximation, the control of effective inductance can be considered as control of a virtual air gap. The virtual air gap (VAG) concept uses the non-linearity of the saturation effect of the core material $[6,7]$. The magnetic core is saturated locally, causing a local decrease of the permeance similar to an inserted air gap.

For standard core geometries this effect can be achieved by biasing currents injected through auxiliary wires, which are inserted via holes in the core branches. The current path is surrounded by a magnetic field, influencing the surrounding ferromagnetic material. Related to the main magnetic flux the additional DC field has the same, opposite or perpendicular direction or any direction in-between. The interactions are usually calculated by numeric field simulation programs [8]. Fig. 2 a) shows the results of the simulation for an example. It is obvious that the width of the core is saturated in a different manner if the locations of the inserted holes are slightly rearranged. The most effective placement of the holes of the design in Fig. 2 a) is horizontally $0.75 \mathrm{~cm}$ with a horizontal distance of $2 \mathrm{~cm}$ and a vertical distance of $1.5 \mathrm{~cm}$ between the holes.
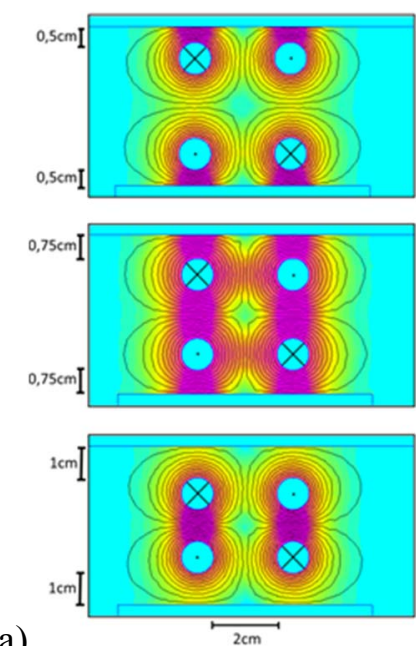

a)
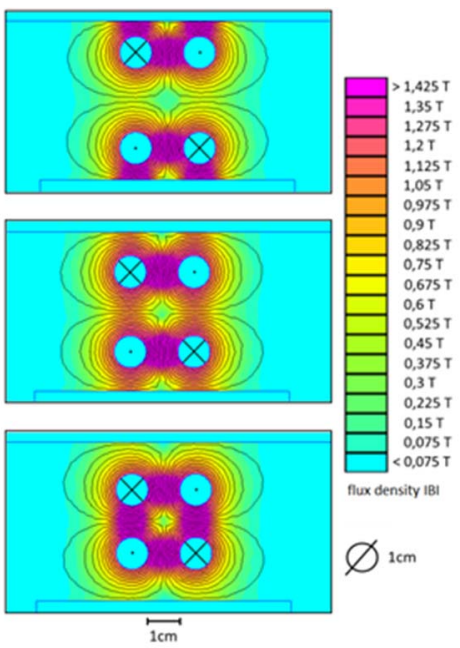

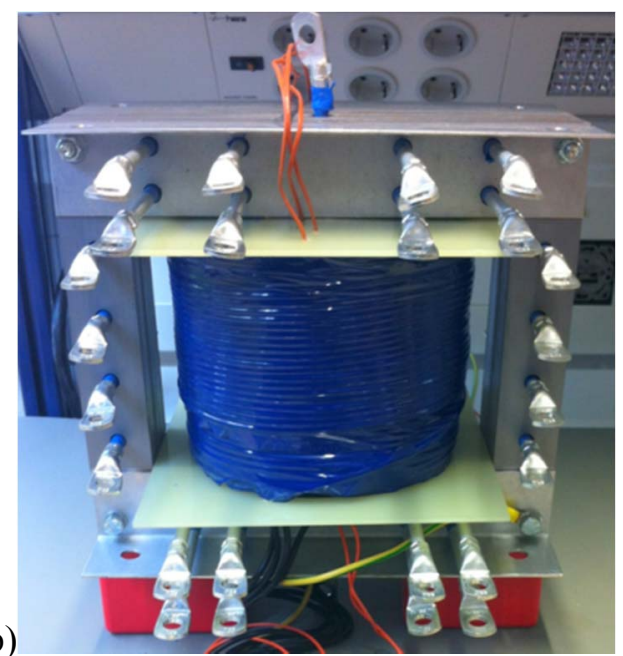

b)

Fig. 2: a) Numerical field simulation of the pre-magnetization field in an upper branch of a magnetic circuit. b) VAG-demonstrator with different pre-magnetization regions 
The concept was evaluated experimentally with the magnetic device shown in Fig. 2 b). Different regions of the core can be biased here. The main excitation winding is applied around the center limb. The biasing is realized with solid aluminum rods. Orthogonal biasing in the center limb is possible, too.

The biasing in direction perpendicular to the main flux in the center leg has a lower effect. This effect can be explained by the fact that the biasing field runs transversely through the metal sheet package. Thus, there are many small air gaps between the sheets avoiding high flux density along the biasing field lines. The biasing in the yoke enabled a recognizable influence on the hysteresis. The most effective change of the hysteresis and the inductance is performed by the biasing of the outer legs.

Fig. 3 a) shows the $\Psi(I)$ curves for the main winding of the investigated demonstrator from Fig 2 b). Obviously, the hysteresis characteristics can be stretched by biasing the different parts of the core. For comparison of the design effectiveness all measurements were performed with the same biasing current. Fig. 3 b) shows the inductance dependent on the ampere turns for different biasing locations as an overview.

a)

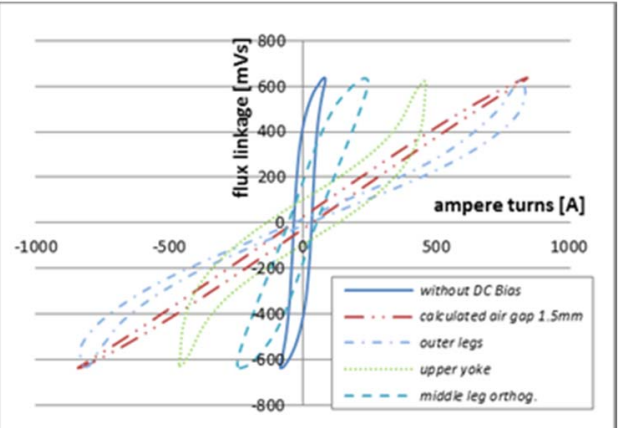

Fig. 3: a) Measured $\Psi(I)$-curves with different biasing locations. b) Inductance vs. DC-bias measurement

\section{Example: design of controllable inductor for reactive power compensation}

Fig. 4 a) shows a controllable reactive power compensation reactor. It consists of a ferromagnetic core, an $\mathrm{AC}$ winding, two DC windings and an air gap. The inserted air gap is used to realize the basic inductance of the device for the case that the reactive current does not have to be adjusted by biasing. For the purpose of designing the variable inductance, the magnetic circuit according to Fig $4 \mathrm{~b}$ ) can be used, where:

$$
\begin{aligned}
& R_{\mathrm{m}}=\frac{l}{\mu \cdot A}=\frac{V_{m}}{\phi}=\frac{N \cdot I_{S}}{\phi} \\
& \psi=\int u_{S}(t) \cdot d t \\
& i_{\mathrm{S}}=\frac{R_{m}}{N^{2}} \cdot \int u_{S}(t) \cdot d t=\frac{1}{L} \cdot \int u_{S}(t) \cdot d t
\end{aligned}
$$

- $R_{\mathrm{m}}=$ reluctance

- $l=$ length

- $A=$ cross section

- $\mu=\mu_{0} \cdot \mu_{\mathrm{r}}=$ permeability

- $N=$ number of turns

- $\phi=$ magnetic flux

- $\psi=$ magnetic flux linkage

- $u_{\mathrm{S}}=$ input voltage

- $i_{\mathrm{S}}=$ input current 


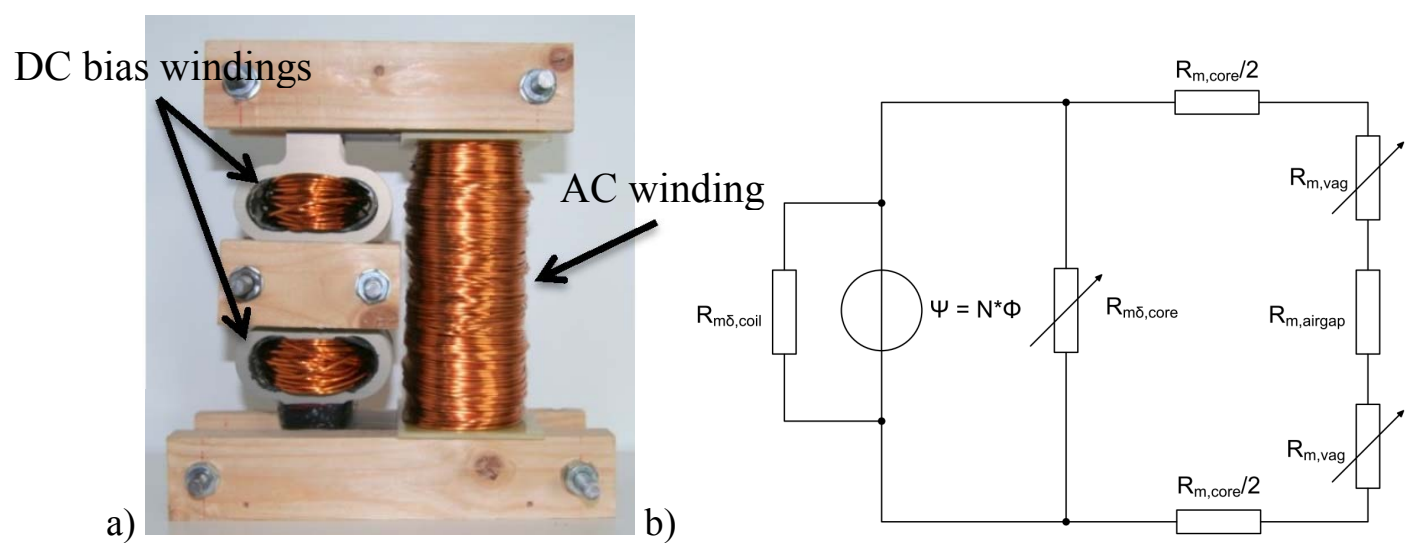

Fig. 4: a) Experimental setup. b) Magnetic circuit

a)

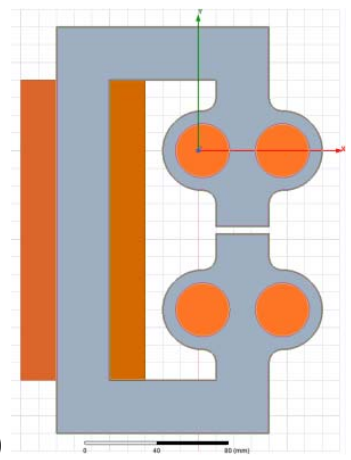

b)

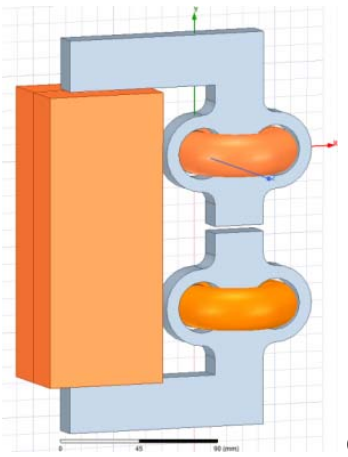

c)

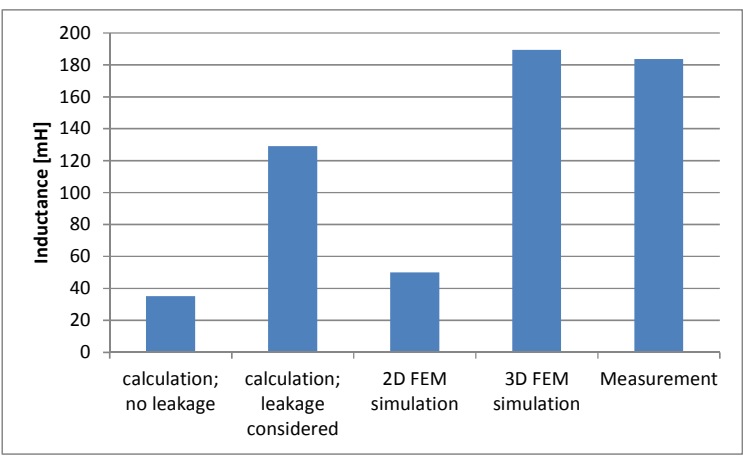

Fig. 5: a) 2-D FEM simulation model. b) 3-D FEM simulation model. c) Evaluated inductance values

The voltage $U_{S}$ is supplied by the connected circuit (e.g. grid). The reactive current $I_{S}$ can be set by changing the total reluctance $\mathrm{R}_{\mathrm{m}}$. The device provides its maximum inductance if no DC bias current is applied. This means the VAG is not active. The reactive current consumption is minimal because the reactance is maximal. In this case the reluctance $R_{m, V A G}$ can be neglected for the design. As a first approach the leakage reluctance $\mathrm{R}_{\mathrm{m}, \delta, \text { core }}$ is neglected as well. These simplifications lead to a series magnetic circuit containing the reluctance of the core and the air gap. The calculation of this magnetic circuit gives an inductance of $35 \mathrm{mH}$. The insertion of the leakage flux of the core into the model according to [9] and the recognition of the air gap fringing flux $\left(\mathrm{R}_{\mathrm{m}, \delta, \text { core }}\right)$ gives a calculated inductance of $129 \mathrm{mH}$.

It should be noted that the model from [9] neglects the different permeability values of the core material and the different magnetization conditions. Thus, this model is only geometry dependent. To check the results of the calculated designs, a numerical field calculation was performed. The 2-D FEM simulation model from Fig. 5 a) provides an inductance of $50 \mathrm{mH}$. In contrast, a 3-D FEM model according to Fig. $5 \mathrm{~b}$ ) provides an inductance of $189.5 \mathrm{mH}$. The different approaches show results in a range of 35 to $189.5 \mathrm{mH}$. The experimental investigation of the reactive power reactor from Fig. $4 \mathrm{a}$ ) showed an inductance of $183.6 \mathrm{mH}$. From this result it becomes clear that the leakage flux leaving the core material is not negligible. The leakage flux depends on the geometry, material and the state of the magnetization. The best way to capture these effects is the implementation of a 3-D field calculation. The calculation of the leakage according to [9] by taking the geometric model into account gives a first approximation. Calculations that neglect the leakage of the device or $2 \mathrm{D}$ simulations can lead to totally wrong inductance values and are not recommended.

\section{Bias winding parallel to the main flux}

\section{Controlled devices based on ferrite materials}

Ferrite materials are applicable for high frequency applications. They provide high magnetic and low electric conductivity. In general, they exhibit low core loss compared to other ferrous materials. In power electronic applications ferrite core inductors are gapped in order to enable the capability to store 
magnetic energy in the device. The inserted air gaps linearize the inductance-current characteristic of the inductor and enable a constant inductance value for the operation range. Operation of the component in the saturation region leads to an inductance roll-off until the minimum inductance value according to the coil geometry is reached.

Generally, the inductance value can be controlled by the measures given in the second section of this paper. The following measures are focused on the virtual air gap theory [10]. Fig. 6 a) shows a pot core inductor with center hole. A wire is carried through the center, enabling a field injection perpendicular to the excitation winding on the center leg. The inductance value of the device can be changed dependent on the DC current flow of the center hole wire. The advantage of the perpendicular biasing is that the magnetic fields excited by the different windings will not disturb the other winding. Fig. $6 \mathrm{~b}$ ) shows the flux linkage vs. ampere turns characteristic of the inductor dependent on different

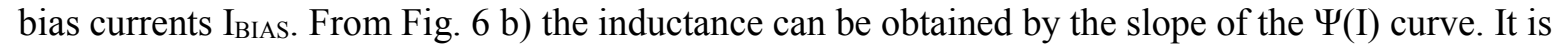
obvious that the inductance of the device is influenced by the perpendicular biasing. Currents up to $20 \mathrm{~A}$ enable only slight changes of the inductance for the demonstrated inductor. Higher bias currents in a range of $40 \mathrm{~A}$ and $80 \mathrm{~A}$ deform the characteristic and lead to a non-linear inductor behavior.

a)

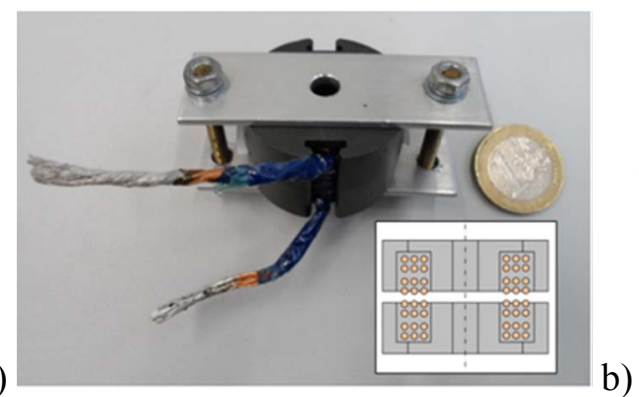

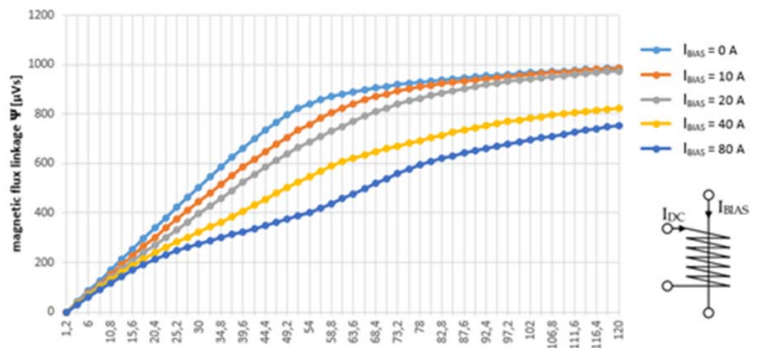

magnetic ampere turns $\theta_{\infty}[\mathrm{A}]$

Fig. 6: a) P36 ferrite core inductor with controllable inductance. b) Flux linkage vs. ampere turns dependent on perpendicular bias current

The bias current is used to partially saturate the core. This measure causes a reluctance change and, therefore, a change of the inductance of the device as an integral parameter. The saturated parts of the core displace the flux excited by the main winding. Fig. 7 shows the simulated saturation of the P36 ferrite core from Fig. 6 a). The bias winding is carried through the center hole and is arranged perpendicular to the main winding. Fig. 7 shows how the saturation inside the core extends if the bias current increases. The center limb of the core starts saturating first. It becomes almost totally saturated even for low bias currents. Higher currents start saturating the top and bottom plates of the core. Very high bias currents enable even the saturation of the outer core segments.
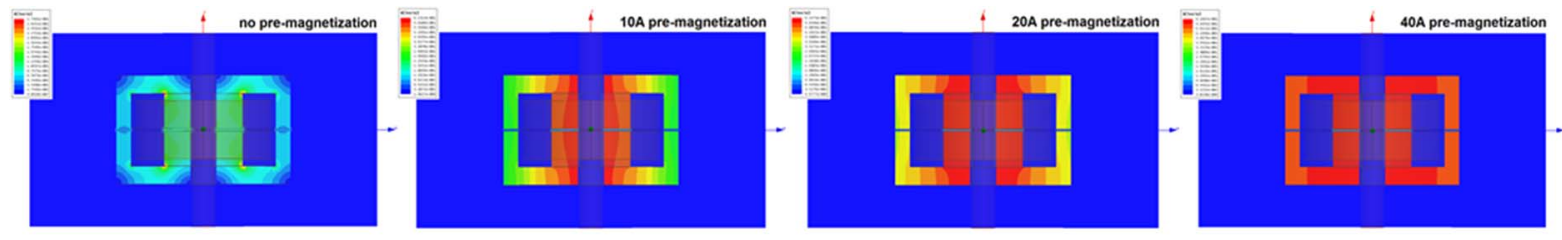

Fig. 7: 3D FEM simulation of flux density distribution inside the core for increasing bias currents

Fig. 8 a) and b) show vector plots of the flux density and the magnetic field strength for an inductor operated without and with perpendicular biasing. The vector plots without biasing (Fig. 8 a)) show the usual inductor behavior. Flux density and magnetic field strength vectors are aligned in parallel. The highest magnetic field strength values occur inside the air gap. If small air gaps are considered, the field inside the gap can be assumed to be almost homogenous. In comparison, the perpendicular biasing deforms the field vectors in direction of the bias field as can be seen in Fig. 8 b). The magnetic fields of both windings overlap and the direction of the total field changes dependent on the direction and the field strength values of the different fields. From the comparison of Fig. 8 a) and b) it becomes obvious, that the direction as well as the length of the magnetic field vectors have been changed caused by the perpendicular biasing. 
This effect explains the inductance change of the device. The inductance value for a given current can be calculated by the magnetic energy mainly stored inside the air gaps of the component. The energy per volume is given by the magnetic field strength and the flux density, where the integration over the total volume gives the magnetic energy of the device in Joules.

a)

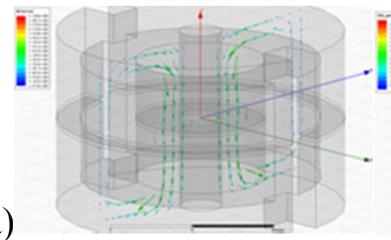

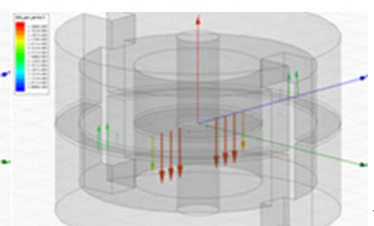

b)

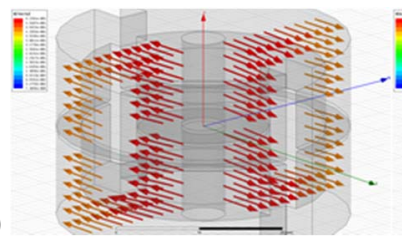

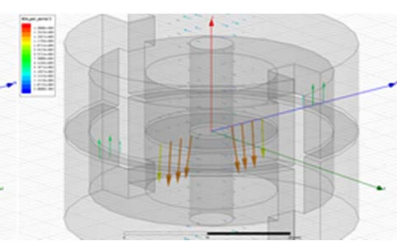

Fig. 8: a) Flux density (left) and magnetic field strength (right) vectors for inductor without biasing. b) Flux density (left) and magnetic field strength (right) vectors for inductor with heavy biasing

The previous example showed that high inductance changes require high biasing ampere turns. In order to reduce the bias current the number of turns of the biasing winding can be increased. This concept was realized for the inductor from Fig. 9 a) showing an example of an orthogonal biased $\mathrm{E}$ core inductor. The yellow bias winding is carried through six holes of the center limb in order to increase the ampere turns of the biasing winding. Fig. $9 \mathrm{~b}$ ) shows the measured hysteresis with and without orthogonal biasing of the center limb. The orthogonal biasing deformed the hysteresis loop of the device. Different slopes can be observed in the flux linkage-current curve. This results in different amplitude inductance values dependent on the operation point as it is the case for Fig. 6 b).

a)

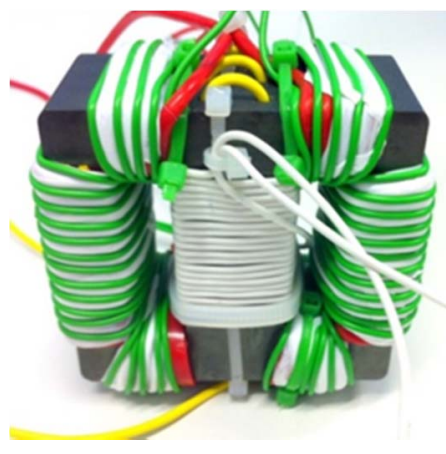

b)

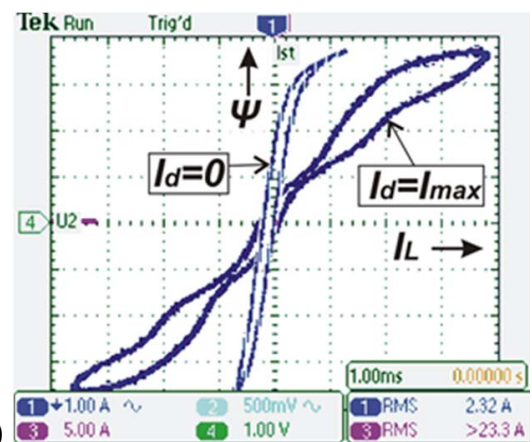

Fig. 9: a) E core inductor with bias (yellow), measurement (white) and main winding (green).

b) Measured hysteresis curve of $E$ core inductor

\section{Modelling of parallel DC biasing controlled devices}

In conventional transductor technology the operation point is shifted by means of a direct current biasing of a magnetic section. This leads to a reluctance change of the magnetic core. For the simulation of the electric behavior including the controlled magnetic branches the "gyrator properties" of an electromagnetic device have to be taken into account. In case of parallel DC biasing a magnetic flux is generated by the direct current of the control winding and is directed in parallel to the magnetic main flux of the inductor. Both magnetic fluxes are either rectified or aligned in opposing direction.

A DC bias controlled inductor can be represented by the electric symbol given in Fig. 10 b). Fig. 10 c) shows an electric circuit model of a controllable inductor or inductor branch, biased by parallel DC flux. The inductor/inductor branch is represented by a linear component $\mathrm{L}_{01}$ and a non-linear component $\mathrm{L}_{1}$. The linear part represents the "air / vacuum fraction" of the magnetic circuit without any ferrous core material. The non-linear part presents the non-linear polarization of the core material. The total voltage drop $U_{\mathrm{L}}$ across the inductor/inductor branch consists of voltage $U_{\mathrm{L} 01}$ along $\mathrm{L}_{01}$ and the induced voltage $U_{\mathrm{L} 1}$ at the non-linear component $\mathrm{L}_{1}$. The model structure is equivalent to a "nonlinear" gyrator. Losses can be included by inserting a non-linear resistance in parallel to $L_{1}$. 


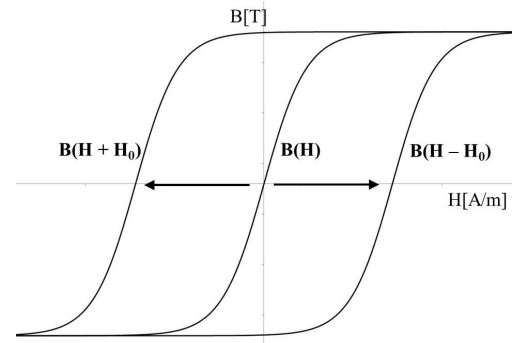

a)

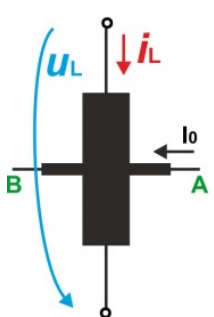

b)

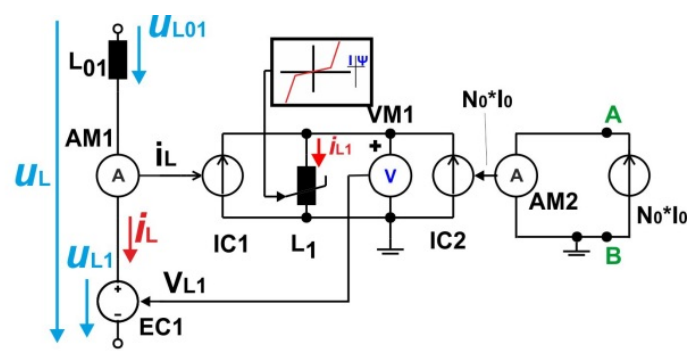

c)

Fig. 10: Magnetic control basics: a) Hysteresis characteristics for parallel DC biasing. b) Electric circuit symbol. c) Modeling of a DC biased inductor by an electric circuit with controlled sources

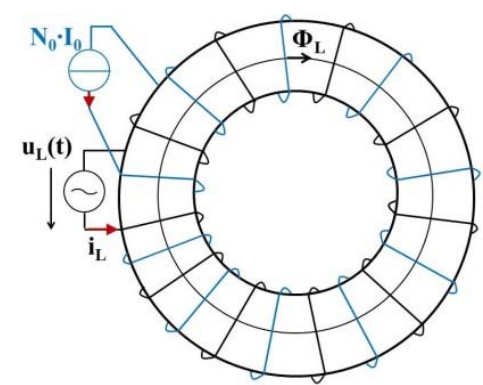

a)

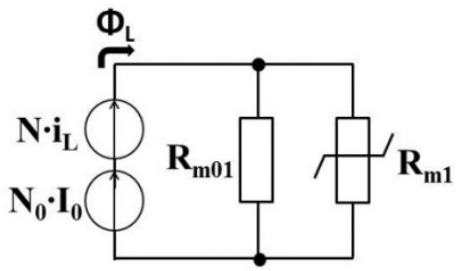

b)

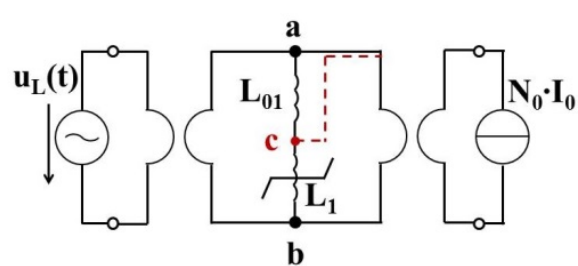

c)

Fig. 11: a) Parallel DC bias controlled inductor. b) Equivalent magnetic circuit of the inductor.

c) Transformed electric circuit

Fig 11 shows an inductor controlled by a parallel DC bias and its equivalent magnetic and electric circuit. The electric circuit of the inductor in Fig. $11 \mathrm{c}$ ) can be converted into the electric circuit model shown in Fig. 10 c). In order to enhance numerical stability of the simulation model the DC magnetomotive force $\mathrm{N}_{0} \cdot \mathrm{I}_{0}$ is connected directly to the non-linear component $\mathrm{L}_{1}$ (pin c-b in Fig. $11 \mathrm{c}$ )) instead of to the linear component $\mathrm{L}_{01}$ together with $\mathrm{L}_{1}$ (pin a-b in Fig. $11 \mathrm{c}$ )). This measure in the model is uncritical because the DC magneto-motive force does not induce any additional voltage.

The effect of the parallel DC biasing in the $\Psi$-I characteristic of the inductor can be simulated by the proposed electric circuit model. The operation principle of a gyrator is implemented in this model. As an advantage a complicated simulation for the magnetic circuit can be replaced by a simple electric model. For the simulation process with the electric model an I- $\Psi$ characteristic of an exemplary soft magnetic material is inserted for the component $\mathrm{L}_{1}$.

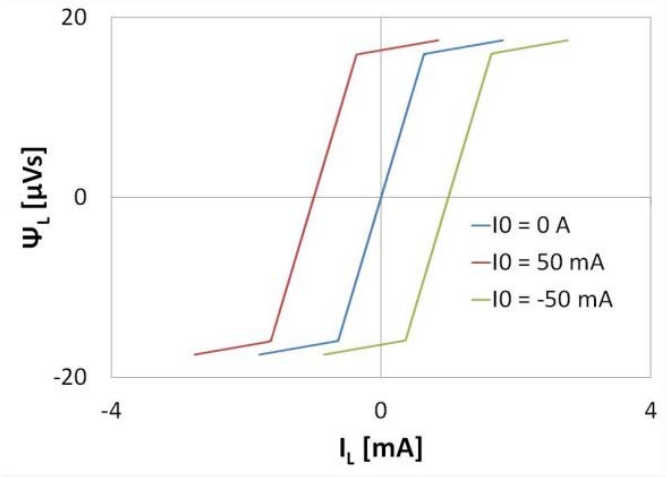

a)

Fig. 12: Simulation results: a) $\Psi-I$ characteristic with different bias currents. b) L-I characteristic with different bias currents

Fig. 12 a) and b) show how the parallel DC biasing affects the $\Psi$-I characteristic as well as the differential inductance by means of exemplary parameters $\left(\mathrm{N}, \mathrm{N}_{0}, \mathrm{I}_{0}\right.$ etc.). 
Fig. 13 represents the measurement results of an AMCC- 63 core with a parallel biasing generated by a permanent magnet. The red measured curve shows the effect of the parallel biasing on the inductance. Both the simulation results based on the theoretical model in Fig. 12 b) and the measurement results in Fig. 13 show the same behavior. Finally, an electrically parallel DC biasing and a parallel bias by a permanent magnet can be implemented with this model.

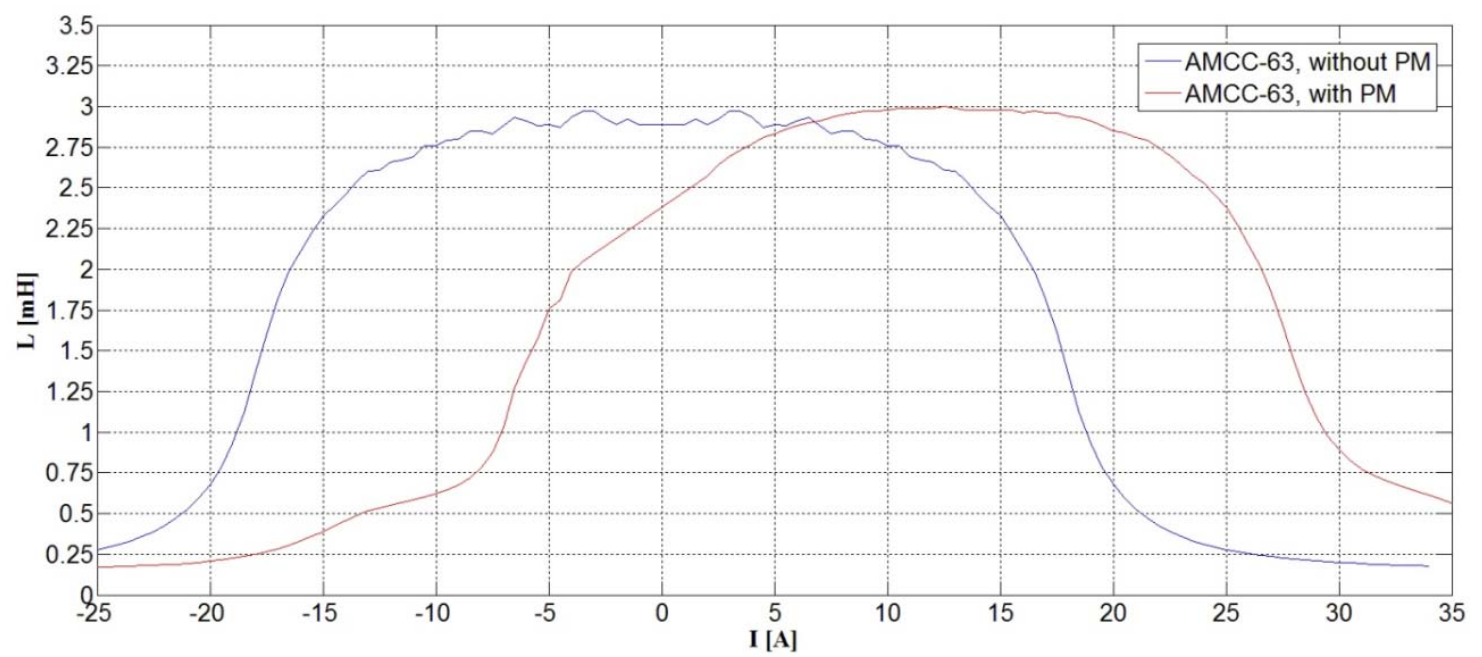

Fig. 13: L-I measurement results of a AMCC-63 core without parallel biasing and with parallel biasing by permanent magnet [11]

The mathematical model shown in Fig. $10 \mathrm{c}$ ) can also be used to simulate orthogonal DC biasing. The effective magnetic field strength can be achieved by local superposition of both magnetic field strengths. In case of isotropic magnetic material the $\mathrm{B}(\mathrm{H})$ characteristic results in an according flux density. The total flux density vector is composed of the different axis coordinate directions of the overlain flux densities. Due to the fact that the magnetic bias field strength in Fig. 1 b) is not homogenous this problem is solved by FEM simulations. The homogenous case can be considered directly in Fig. 10 c) by a few changes of the controlled current source IC2. These aspects can be considered in future developments.

\section{Summary}

The paper shows several approaches how to change and control the reluctance of branches in magnetic circuits by electrical biasing methods. Some advantages and disadvantages of the different methods were highlighted. The impact of the biasing on the inductance value depends on many factors such as the implementation and arrangement of the biasing winding as well as on geometry and material parameters. The parallel biasing allows high changes of the inductance characteristic but causes issues regarding unwanted magnetic coupling. Other techniques working with biasing windings in parallel or orthogonal to the main flux may require high ampere turns in order to enable high inductance changes. This can be achieved by inserting multiple holes into the magnetic core in order to enable more biasing winding turns.

The possibility of controlling inductive devices offers new design options in power electronics in both high power and high frequency application. Controllable inductors and transformers are interesting for applications such as reactive power compensation, resonance converters and other applications that require variable storage or filter inductors and transformers. For example an adaption of the choke inductance during operation is as possible as the change of the voltage transformation ratio of a transformer. However, more detailed investigations regarding the suitability of the different biasing methods have to be done in future in order to bring controllable inductive devices effectively into service.

A further aspect is the trend of the demand for higher degrees of component integration and power density of power electronic devices and converters. The implementation of high integrated inductive devices can enable both high power densities and efficiency values [12]. The question how techniques 
of high integrated magnetic components and controllable devices can be combined is not answered yet though basics for both techniques are known. Nevertheless, the modelling of controllable inductive devices requires further research. The modelling of parallel DC biasing is possible even with simple electrical circuit models, whereas the reliable investigation of bias windings in parallel or orthogonal to the main flux require still the implementation of 3D FEM models. Further improvements in modelling controllable devices will enhance the design and help to transfer the technique into applications.

\section{References}

[1] Platt S.: Magnetic Amplifiers - Theory and Application, Prentice Hall, 1958, 254pp.

[2] Friebe J. and Zacharias P.: Review of Magnetic Material Degradation Characteristics for the Design of Premagnetized Inductors, IEEE TRANSACTIONS ON MAGNETICS, VOL. 50, NO. 3, March 2014

[3] Stadler A.: Messtechnische Bestimmung und Simulation der Kernverluste in weichmagnetischen Materialien, Dissertation University of Erlangen-Nürnberg, 2009

[4] Medini D. and Ben-Yaakov S.: A Current-Controlled Variable-Inductor for High Frequency Resonant Power Circuits, Applied Power Electronic Conference and Exposition (APEC), 1994

[5] Hu Y., Huber L. and Jovanović Milan M.: Universal-Input Single-Stage PFC Flyback with Variable Boost Inductance for High-Brightness LED Applications, Applied Power Electronic Conference and Exposition (APEC), 2010

[6] Avila-Montes J., Campos-Gaona D., Vázquez E. M., Rodríguez-Rodríguez J. R.: A Novel Compensation Scheme Based on a Virtual Air Gap Variable Reactor for AC Voltage Control, IEEE TRANSACTIONS ON INDUSTRIAL ELECTRONICS, VOL. 61, NO. 12, DECEMBER 2014

[7] Dolan D. S. L. and Lehn P.W.: Analysis of a Virtual Air Gap Variable Reactor, IEEE Power Electronic Specialists Conference (PESC), 2007

[8] Dolan D. S. L. and Lehn P.W.: Finite element analysis of a Virtual Air Gap Variable Transformer, IEEE Electrical and Computer Engineering, CCECE 2008

[9] Kock J. and Ruschmeyer K.: Permanentmagnete I - Valvo Unternehmensbereich Bauelemente der Philips GmbH, Hamburg, $2^{\text {nd }}$ edition, 1983

[10] Dolan D. S. L. and Lehn P.W.: Finite element analysis of a Virtual Air Gap Variable Transformer, IEEE Electrical and Computer Engineering, CCECE 2008

[11] Friebe J.: Permanentmagnetische Vormagnetisierung von Speicherdrosseln in Stromrichtern, Dissertation University of Kassel, 2014

[12] Kleeb T.: Investigation on Performance Advantage of Functionally Integrated Magnetic Components in Decentralised Power Electronic Applications, Dissertation University of Kassel, 2016 